BULLETIN OF THE

AMERICAN MATHEMATICAL SOCIETY

Volume 81, Number 3, May 1975

\title{
A COUNTEREXAMPLE IN SHAPE THEORY 1
}

\author{
BY JOSEPH L. TAYLOR
}

Communicated by W. Wistar Comfort, December 2, 1974

In the process of trying to solve a certain problem in Harmonic analysis (cf. [5]) the author encountered the following shape theory question: If $f$ : $X \rightarrow Y$ is a surjective map of compact spaces and $f^{-1}(y)$ has trivial shape for each $y \in Y$, then is $f$ necessarily a shape equivalence? We were able to prove that the answer is yes provided $Y$ is finite dimensional, but this was of no use in the problem we were considering.

We discussed this question with R. D. Anderson, who pointed out that the problem has been known to specialists in shape theory (e.g., Anderson, Borsuk, Mardešić) for some time. Anderson has a proof (as yet unpublished) in the case where $\left\{y: f^{-1}(y)\right.$ is nondegenerate $\}$ is finite dimensional. In case $X$ and $Y$ are both finite dimensional the result is essentially Theorem 11 of Sher's paper [4].

The purpose of this paper is to present a counterexample for the general question. Specifically, if $Q$ is the Hilbert cube, we will construct a compact metric space $X$ and a surjective map $f: X \rightarrow Q$ such that $f^{-1}(q)$ has trivial shape for all $q \in Q$, but $X$ does not have trivial shape. Since $Q$ has trivial shape, this means that $f$ is not a shape equivalence.

Our example depends heavily on a $K$-theory result of Adams. In fact, given Adams' result, ours is little more than an observation.

1. Shape. Borsuk [2] invented the concept of shape as a substitute for homotopy type when one is dealing with spaces which are not locally nice (also see [3]). We will not define the notion of shape equivalence here. For our purposes it is enough to know the following: If $f: X \rightarrow Y$ is a continuous map between compact Hausdorff spaces, then $f$ is a shape equivalence if and only if $f^{*}:[Y, M] \rightarrow[X, M]$ is bijective whenever $M$ is an ANR. Thus, if $H$ is any contravariant functor from compact spaces to sets which has a

AMS (MOS) subject classifications (1970). Primary 57A20, 57A60; Secondary $54 \mathrm{G20}, 54 \mathrm{C5} 5$.

1 Research supported by the National Science Foundation under NSF grant \#GP-43114X. 
classifying space which is an ANR, then $f^{*}: H(Y) \rightarrow H(X)$ is bijective if $f$ : $X \rightarrow Y$ is a shape equivalence. Since Čech cohomology and $K$-theory are such functors, a map which is a shape equivalence induces isomorphisms of Čech cohomology and of $K$-theory.

A space $X$ has trivial shape if the injection of a point into $X$ is a shape equivalence. A fact we shall need which is easy to prove is the following: If $X=\varliminf_{\leftarrow} X_{\alpha}$ is an inverse limit of compact spaces and each bonding map $X_{\beta}$ $\rightarrow X_{\alpha}$ is null homotopic, then $X$ has trivial shape.

2. The example. According to Theorem 1.7 of Adams' paper [1], there exists a compact space $F\left(F=S^{2 q-1} \cup_{g} I^{2 q}\right.$ for large $q$ and $g: S^{2 q-1} \rightarrow$ $S^{2 q-1}$ a map of degree $p$ for $p$ an odd prime) and a map $h: S^{r} F \rightarrow F$ such that $h^{*}: \widetilde{K}(F) \rightarrow \widetilde{K}\left(S^{r} F\right)$ is an isomorphism. Here $S^{r} F$ is the $r$ th suspension of $F, r=2(p-1), \widetilde{K}$ is reduced complex $K$-theory, and $\widetilde{K}(F)=Z_{p}$ is nontrivial.

By taking iterated $r$ th suspension of the map $h$ we obtain an inverse limit system

$$
\ldots \rightarrow S^{n r} F \stackrel{S^{(n-1) r} h}{\longrightarrow} S^{(n-1) r} F \rightarrow \ldots \rightarrow S^{2 r} F \stackrel{S^{r} h}{\longrightarrow} S^{r} F \stackrel{h}{\longrightarrow} F
$$

which has a limit $X=\lim S^{n r} F$ with nontrivial $K$-theory $\left(\widetilde{K}(X)=Z_{p}\right)$ and, hence, nontrivial shape. However,

THEOREM. There is a surjective map $f: X \rightarrow Q$ with $f^{-1}(q)$ having trivial shape for each $q \in Q$. Here $Q$ is the Hilbert cube, which obviously has trivial shape.

Proof. The $m$ th suspension $S^{m} F$ of $F$ can be regarded as the space obtained from $I^{m} \times F$ by identifying $\{t\} \times F$ to a point for each $t \in \partial I^{m}$. Hence, there is a surjection $f_{m}: S^{m} F \rightarrow I^{m}$ with $f_{m}^{-1}(t)$ a point if $t \in \partial I^{m}$ and $f^{-1}(t) \simeq F$ if $t \in I^{m} \backslash \partial I^{m}$.

Consider the commutative diagram

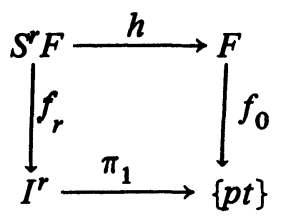

On passing to the cartesian product with $I^{r}$ we obtain a commutative diagram 


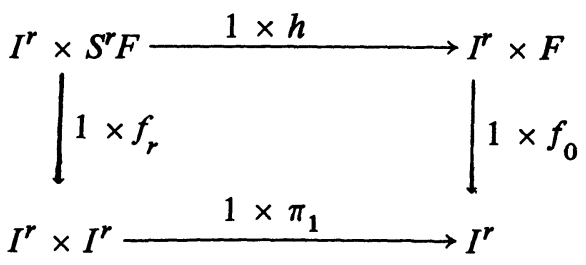

If we collapse fibers of $1 \times f_{r}$ and $1 \times f_{0}$ over points of $\left(\partial I^{r}\right) \times I^{r}$ and $\partial I^{r}$, respectively, we obtain a commutative diagram

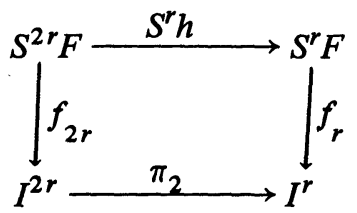

where $\pi_{2}=1 \times \pi_{1}$. Obviously, we can iterate this procedure to obtain a commutative system

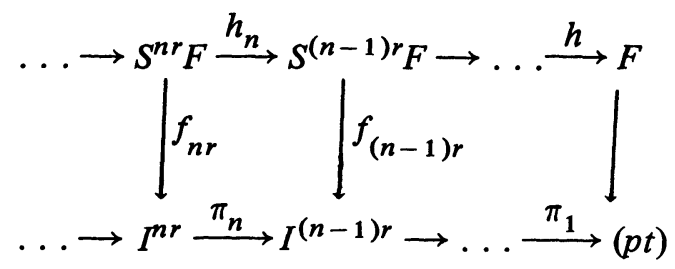

where $h_{n}=S^{(n-1) r} h$. The vertical maps are all surjective and their limit is a surjective map $f: X \rightarrow Q$ where $X=\lim _{n} S^{n r} F$ and $Q=\lim _{n} I^{n r}$ is the Hilbert cube.

$$
\text { If } t=\left\{t_{i}\right\} \in Q \quad\left(t_{i} \in I^{i r}, \pi_{i} t_{i}=t_{i-1}\right) \text { then } f^{-1}(t)=\lim _{n} f_{n r}^{-1}\left(t_{n}\right)
$$

where the $n$th bonding map is the map $k_{n}: f_{n r}^{-1}\left(t_{n}\right) \rightarrow f_{(n-1) r}^{-1}\left(t_{n-1}\right)$ given by restricting $h_{n}$ to $f_{n r}^{-1}\left(t_{n}\right)$.

Now each $k_{n}$ is null homotopic. In fact, if $t_{n} \in \partial I^{n r}$ then $f_{n r}^{-1}\left(t_{n}\right)$ is a point. If $t_{n} \notin \partial I^{n r}$ then

$$
h_{n} \mid f_{n r}^{-1} \pi_{n}^{-1}\left(t_{n-1}\right): f_{n r}^{-1} \pi_{n}^{-1}\left(t_{n-1}\right) \rightarrow f_{(n-1) r}^{-1}\left(t_{n-1}\right)
$$

is a copy of the map $h: S^{r} F \rightarrow F$ and $k_{n}$ is a copy of $h$ restricted to $f_{1}^{-1}(s)$ for some $s \in I^{r}$. The restriction of $h$ to $f_{1}^{-1}(\gamma)$ for $\gamma$ an arc from $s$ to $\partial I^{r}$ yields a homotopy joining $h_{\mid f_{1}^{-1}(s)}$ to a constant map and, hence a homotopy from $k_{n}$ to a constant map.

Since $f^{-1}(t)$ is an inverse limit with null homotopic bonding maps, it 
follows that $f^{-1}(t)$ has trivial shape for each $t \in Q$.

Note that, in this example, each $f_{n r}^{-1}\left(t_{n}\right)$ is either a point or a copy of the finite dimensional space $F$. Hence, the fibers $f^{-1}(t)$ are of bounded dimension. In other words, the problem derives from the infinite dimensionality of the image space $Q$ and has nothing to do with the dimensions of the fibers.

ADDED IN PROof. R. D. Anderson, R. Edwards and J. Keesling (in chronological order) have each written to us pointing out that our example can be used to construct a cell-like map of the Hilbert cube onto a space which is not an absolute retract.

\section{REFERENCES}

1. J. F. Adams, On the groups $J(X)$. IV, Topology 5 (1966), 21-71; errata, ibid. 7 (1968), 331. MR 33 \#6628; 37 \#5874.

2. K. Borsuk, Concerning the notion of the shape of compacta, Proc. Internat. Sympos. on Topology and its Applications (Herceg-Novi, 1968), Savez Društava Mat. Fiz. i Astronom., Belgrade, 1969, pp. 98-104. MR 43 \#1138.

3. S. Mardešić and J. Segal, Shapes of compacta and ANR-systems, Fund. Math. 72 (1971), 41-59. MR 45 \#7686.

4. R. B. Sher, Realizing cell-like maps in Euclidean space, General Topology and Appl. 2 (1972), 75-89. MR 46 \#2683.

5. J. L. Taylor, Homotopy invariants for Banach algebras, Proc. Internat. Congress Math. (Vancouver, 1974), Amer. Math. Soc., Providence, R. I. (to appear). 